\title{
ÉLECTRICITÉ
}

\section{Sur la mise en parallèle Automatique des Machines synchrones et des Centrales électriques}

Les conditions économiques actuelles nécessitent, dans toute exploilation de centrale électrique, la réduction des frais généraux au strict minimum et expliquent l'adoption de plus en plus générale d'appareils automatiques. En effet, l'emploi de régulateurs de tension, de dispositif's automatiques de protection et de démarrage, permet non seulement de réduire sensiblement le personnel de surveillance, mais encore, grâce à la régularité de fonctionnement de ces appareils, d'éviter les fausses manocuvres et leurs conséquences toujours préjudiciables aux machines. Il en résulte une réduction considérable et rapidement sensible des frais de réparation et de renouvellement du matéries.

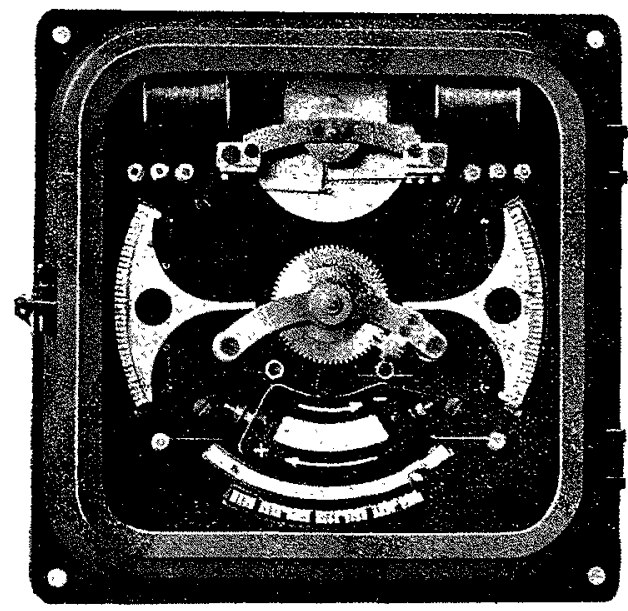

Fig. 1. - Appareil de mise en parallèle automatique type P 2/1. (Système Brown-Boveri).

L'appareil de mise en parallele automatique, que nous allons décrire, répond, par excellence, à ces deux qualités. D'un fonctionnement rapide et sûr, il réalise automatiquement la fermcture de l'interrupteur au moment propice et la personne la plus inexpérimentée ne peut provoquer une mise en parallèle inexacte. Aussi, bien qu'il ne soit que depuis peu de temps sur le marché, est-il déjà d'un usage très répandu (1).

(1) Des appareils de mise en parallèle automatique sont installés dans notre région à :

Manufacture de Glaces et de Produits Chimiques de Saint-Gobain; Centrale d'Avricux (Savoic), sur quatre alternateurs de $3.500 \mathrm{kvA}, 10.000$ volts.

Etablissements Bouchayer et Viallet, Grenoble, mise en parallèle automatique de 2 arrivées du Drac Romanche avec le réseau de la Société de Fure et Morge et de Vizille.

papeteries Peyron et $\mathrm{C}^{10}$, à Vizille, mise en parallèle automatique d'une arrivée de l'usiné de Sćchiliemne avec l'usine génératrice de VizillArticle published by SHF and available at http./WWW sht-mb org or nttp

\section{Mode de Fonctronneneyt}

L'appareil de mise en parallèle automatique se branche comme les lampes de phase et autres dispositifs de synchronisation, sur les deux tensions individuelles des groupes à mettre en parallèle (voir schéma fig. 3). Il se compose essentiellement d'un relais à action différée à contacts de fermeture dépendant d'un dispositif modificateur particulier caractérisant l'appareil. Ce relais, basé sur le principe de Ferraris, est constitué par un disque d'aluminium, supporté par deux pointes en acier, qui, sous l'influence du champ tournant créé par deux électro-ai mants alimentés respectivement par chacune des tensions des deux groupes à coupler, enroule un cordonnet dont l'extrémité est fixée à un contact à ressort. Ce dernier, si les bobines des électro-aimants restent un temps suffisant sous tension, vient s'appliquer contre un contact fixe et établit le circuit entre les bornes 9 et 10. Le relais commandant la bobine d'enclenchement de l'interrupteur est alors actionné et provoque la fermeture de ce dernier et par suite la mise en parallèle.

Les enroulements du relais à action différée sont connectés aux tensions individuelles de telle sorte que, lorsque les groupes à mettre en parallèle sont en phase, la tension totale aux extrémités des enroulements est le double de chaque tension indivi-

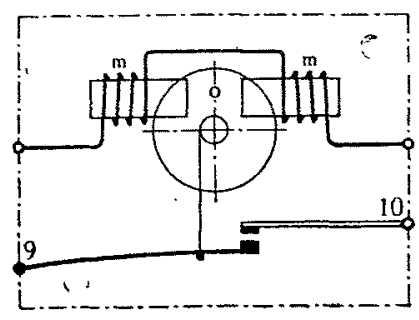

Fig. 2. - Relais à action différée de l'appareil de mise en parallèle.

duelle (couplage à l'allumage des lampes). La figure 4 représente le diagramme des variations de la tension aux extrémités des bobines et par conséquent du courant passant par ces bobines. Ce diagramme est établi en supposant que les valeurs des fréquences respectives des deux groupes, d'abord assez différentes, se rapprochent de plus en plus provoquant une durée de plus en plus importante des périodes d'interférence. La différence angulaire de phase des deux groupes est indiquée en degrés sur l'abscisse ; pour une différence angulaire de $180^{\circ}$, le courant est égal à zéro, et il atteint son maximum pour une différence angulaire égale à zéro, c'est-à-dire à l'identité de phase et repasse de noud产. 
$180^{\circ}$. Si l'on suppose maintenant le relais réglé pour un courant $J r$, le disque d'aluminium commence à tourner dès que le courant coupe la ligne $J_{I}$, c'est-à-dire aux points $c 1, c 2$, $c 3$ et $c 4$ des périodes d'interférence. Le courant diminuânt de nouveau le disque s'arrètera lorsque la ligne $J r$ sẹra de nouveau traversée, c'est-à-dire aux points $d 1$, $d 2$ et $d 3$; puis, sous l'influence du ressort du contact, un mouvement de retour se produira provoquant le déroulement du cordonnet.

Afin que le contact püisse se fermer, il faut que le, disque enroule le cordonnet pendant une durée égale à ca. Pour les trois premières périodes d'interférence, ceci ne se produit pas, car les points $a$ 2 et $a 3$, par exemple, où la fermeture du contact pourrait avoir lieu, sont déjà en dehors de la courbe du courant et le disque a déroulé de nouveau le cordonnet. Ce n'est qu'à la quatrième période d'interférence, au point $a 4$, que le cordonnet est complètement enroulé et que le contact se ferme. La commande à distance entre en action et, environ au point $b$, produit la fermeture de l'interrupteur à huile. Les deux groupes sont alors couplés en parallèle et le courant, retombé en partie, remonte immédiatement à sa valeur maximum pour y rester jusqu’à ce

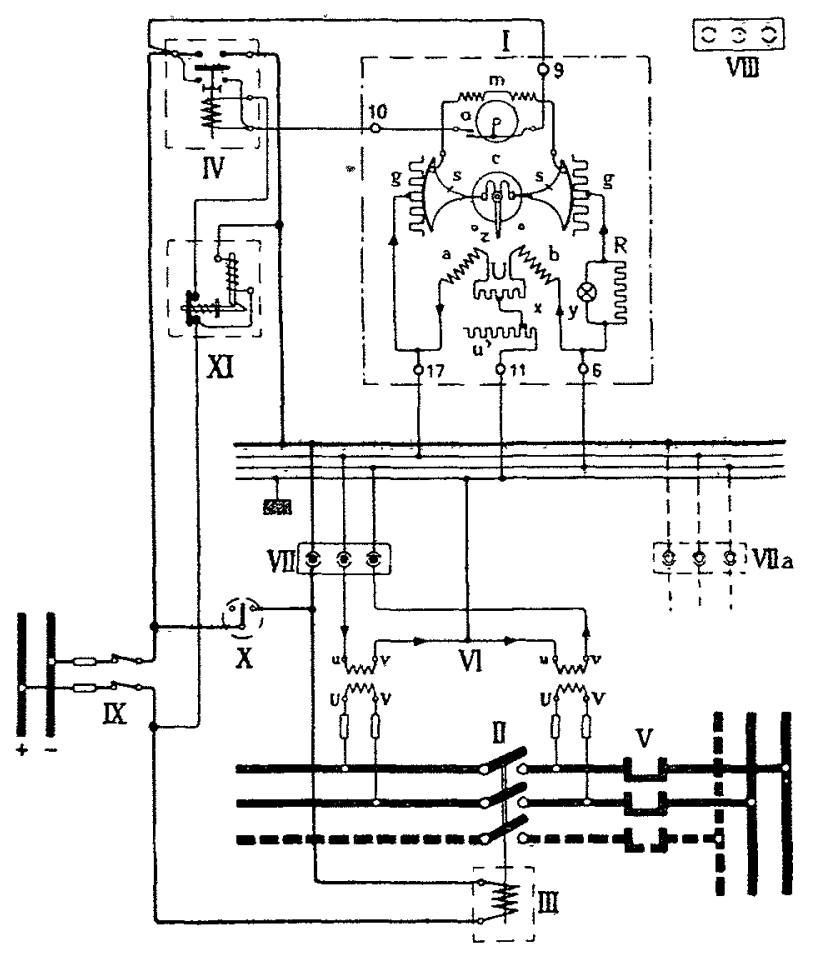

Fig. 3. - Disposition de l'appareil de mise en parallèle automatique pour une installation triphasée (commande à distance avec contact de fin de course).

$\mathrm{I}=$ Appareil de mise en parallele automatique.

II $=$ Interrupteur $\dot{a}$ huile.

III = Commande à distance.

IV = Relais intermédiaire avec bobine d'arrêt.

$\mathrm{V}=$ Sectionneur.

$\mathrm{VI}=$ Transformateur de tension.

VII-VII a $=$ Commutateurs à fiche pour les interrupteurs à huile.

VIII $=$ Boite à fiche vide.

$\mathrm{IX}=$ Interrupteur du circuit auxiliaire.

$\mathrm{X}=$ Commutateur à thain de la commande à distance.

$\mathrm{XI}=$ Interrupteur pour le relais intermédiaire.

que, l'appareil de mise en parallèle déconnecté à la main, le circuit du relais soit interrompu.

En examinant la figure 4, on remarquera que la mise en parallèle n'aurait pas lieu exactement à l'instant où se produit l'iden- tité de phase, c'est-à-dire au moment où le courant du relais atteint sa valeur maximum, mais avec un certain retard. II se produirait done un courant d'échange entre les deux groupes qui pourrait atteindre une valeur importante et par conséquent dangereuse pour l'installation.

Le dispositif modificateur a pour but d'éliminer ce retard ; it cet effet, les bobines du relais à action différée ne sont pas branchées directement sur les deux tensions individuelles, mais pạr l'intermédiaire de résistances de régulation $g$. Ces résistances sont composées d'éléments réunis chacun à un plot de contact. Sur ces plots, disposés en arcs de cercle, roulent des secteurs de contact $s$, guidés par leur pointe en acier reposant sur une crapaudine à pierre fine évidée reliée par un ressort au tambour

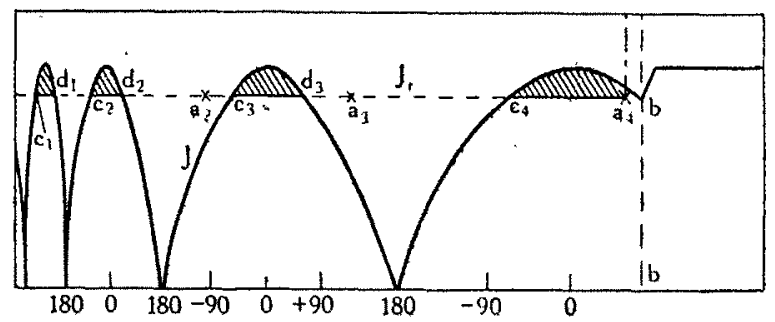

Fig. 4. - Diagramme du courant passant par les bolsines du relais $\hat{a}$ action différée (sans l'influence du dispositif modilicateur).
$I=$ Courbe du courant.
$J^{r}=$ Intensite du fonctionmement du relais a action ditléréc.
a = Instant de la fermeture du contact.
$b_{*}=$ Instant de la fermeture complete de l'interrupteur a luile.

tournant $c$. Les mouvements de rotation de ce tambour se transmettent ainsi aux secteurs et provoquent le déplacement du point de contact avec les plots.

Ce dispositif bien connu est employé depuis longtemps avec snccès sur les régulateurs à action rapide (1).

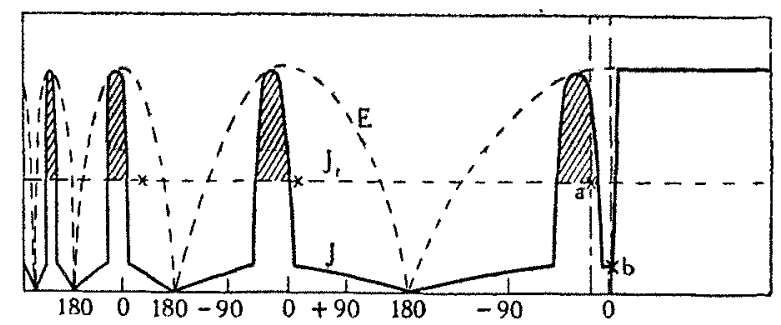

Fig. 5. - Diagramme du courant passant par les bobines du relais à action différée (sous l'influence du dispositif modificateur).

$$
\begin{aligned}
& \mathrm{E}=\text { Courbe de la tension aux bornes de lapparell. } \\
& \mathrm{J}=\text { Courbe du courant. } \\
& \mathrm{Jr}=\text { Intensite du conetionnement du relais a action différée. } \\
& \mathrm{a}=\text { Instant de la fermeture du contact. } \\
& \mathrm{b}=\text { Instant de la fermeture complete de l'interp̧upteur a hule. }
\end{aligned}
$$

Les mouvements du tambour sont provoqués, d'après le principe Ferraris, par un système d'électro-aimants dont les enroulements $a$ et $b$ sont combinés avec les résistances auxiliaires $U$

(1) Voir Houlle Blanche, janvier à octobre 1922.

Des diverses applications du régulateur à action rapide "BrownBoveri " et des methodes de branchement correspondantes (V. Sylvestre). 
el $u^{\prime}$ de façon à produire un chanlp Lournant. Le couple auquel est soumis le tambour sous l'eflet de ce champ tournant est nul quand il y a identité de phase cntre les deux groupes à mettre on parallèle; par contre, dès qu'il y a une différence angulaire, le tambour tourne dans une direction ou dans l'autre jusqu'à ce qu'il soit arrêté par les butées limitant sa course. Le mouvement des secleurs provoquant un déplacement du point de contact sur les plots modifie la résistance insérée dans le circuit du relais à action différée. Quand le tambour est dans sa position d'arrêt au milieu de sa course, c'est-à-dire dans le cas d'identité de phase des deux unités à mettre en parallèle, les résistances $g$ sont courtcircuitées, et le relais à action dilférée, est soumis à la valeur totale des deux tensions individuelles; pour toutes les autres positions du tambour, une fraction de résistance est insérée dans le circuit ef absorbe une partie de la tension; lorsque le tambour

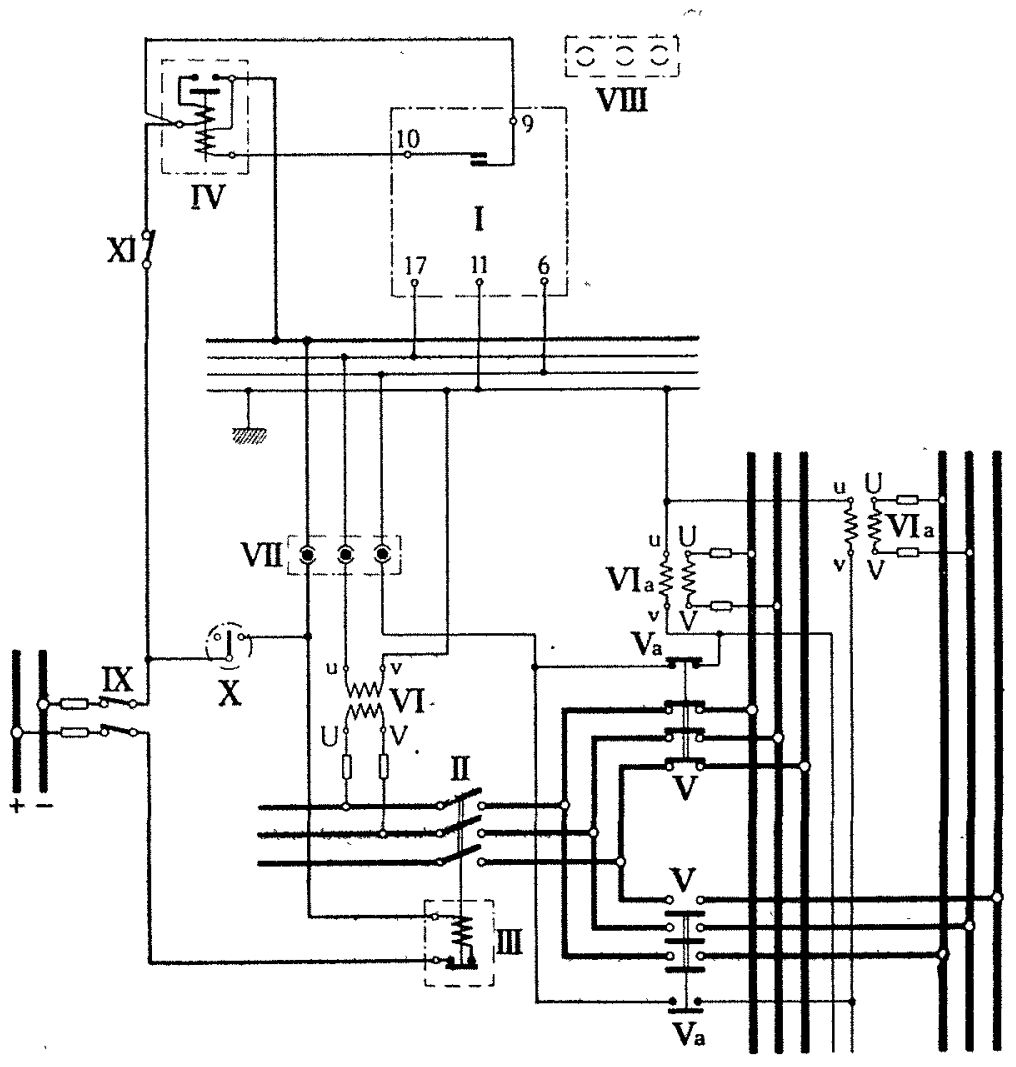

Iنig. 6. - Disposition de l'appareil de mise en parallèle automatique pour tno installation triphasée aved plusieurs systèmes de barres (contacts de verrouillage sur les sectionneurs).

$$
\begin{aligned}
& I=\text { Appareil de mise en parallèle antomatique. } \\
& \text { II }=\text { Interruptenr } \dot{a} \text { buile. } \\
& \text { III }=\text { Commande it distance. } \\
& \text { IV }=\text { Relais intermédiaire avee bohine d'arret. } \\
& \mathrm{V}=\text { Sectionneur. } \\
& \mathrm{V} \mathrm{a}=\text { Contact de verrouillage. } \\
& \mathrm{VI}=\text { Transformateur de tension de l'alternateur. } \\
& \text { VI } \mathbf{a}=\text { Transformateur de tension des barres. } \\
& \text { VII-VII } \mathrm{a}=\text { Commutateurs à fiche pour les interrupteurs à húile. } \\
& \text { VIII }=\text { Boile a fiche vide. } \\
& \mathrm{IX}=\text { Interrupteur du circuit auxiliaire. } \\
& \mathrm{X}=\text { Commutateur ì main de la commande it distance. } \\
& \mathrm{XI}=\text { Interrupteur pour le relais intermédiairc. }
\end{aligned}
$$

est dans une de ses positions extremes, la plus grande partio de la tension est absorbee dans les résistances et le courant passant par le relais à action différéc est très faible.

Le diagramme de la figure 5 , montre clairement l'effet de cette résistance variable sur le courant du relais. Si ce dernier est réglé pour un courant $J r$, lo courant passant par les bobines sera pen- dant la plus grande partie de la période d'interférence bien inférieure à $J r$; ce n'est qu'aux environs immédiats de l'identité de phase que le courant augmentera brusquement; le disque du relais subira à ce moment une impulsion énergique provoquant un enroulement rapide du cordonnet, et, par suite, la fermeture du contact. Il est évident que la forme de la courbe du courant de la figure 5 correspond à une précision de fonctionnement du relais bien plus grande que celle de la figure 4 .

La liaison entre les secteurs de contact et le tambour tournant n'est pas rigide. Elle est constituée par un accouplement à friction. La course des secteurs est limitée, comme celle du tambour tournant, par des butées; toutefois la distance entre les butées du tambour correspond à un angle plus ouvert que celle des butées des secteurs; il en résulte qu'à chaque oscillation, les secteurs sont arrêtés avant le tambour. Le couple agissant sur le tambour est suffisant pour vaincre la friction "de l'accouplement, de sorte que le tambour continue son chemin provoquant un décalage angulaire des contacts par rapport à ce dernier. Ce décalage angulaire se produit en sens opposé, suivant que les secteurs ont buté à gauche ou à droite, mais dans les deux cas, il est tel que lorsque le point de contact passe par la position mé-

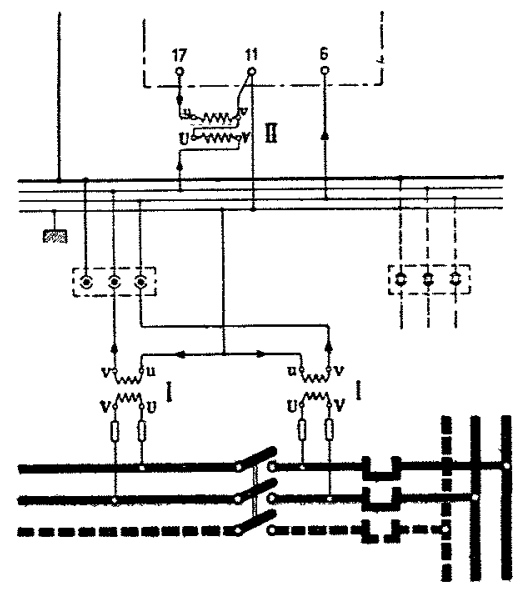

Fig. 7. - Disposition du transformateur-inverseur pour modifier le couplage à l'extinction en couplage à l'allumage des lampes.

$$
\begin{aligned}
I & =\text { Transformateur de tension existant. } \\
\text { II } & =\text { Transformateur inverseur. }
\end{aligned}
$$

cliane il est en avance par rapport au tambour. Le point de contact atteindra donc la position médiane avant l'identité de phase des deux groupes et, de même, provoquara le court-circuitage des résistances $g$ avec une certaine avance.

Ceci est clairement indiqué sur la figure 5 , où la valeur maximum du courant $J$ est en avance par rapport à la valeur maximum de la tension $E$. Le résultat pratique de ce décalage est évident : le contact de l'appareil de mise en parallele automatique provoquera l'enclenchement de l'interrupteur à huile assez tòt pour que, malgré le retard causé par lâ commande à distance de cet interrupteur, la mise en parallèle des deux groupes ait lieu aussi exactement que possible au moment de ridentité de phase.

La distance dos butées et par conséquent le décalage peuvent être réglés de façon que le retard, variable avec les différents systèmes de commandes à distance, soit compensẻ exactement.

Un contact de réglage, pouvant ètre déplacé à la main sur les plots de la résistance $u$ ' permet d'influencer le couple du tambour 
$c$; de celte façon, on peut augmenter ou diminuer sensiblement la précision de fonctionnement de l'appareil. Si le contact de réglage est poussé jusqu'à la position extrême gauche, la mise en parallèle n'est pas rigoureusement exacte, mais alors elle est obtenue très rapidement. Ceci est indispensable lorsqu'il s'agit de services présentant des à-coups de charges brusques et violents, car alors les fréquences respectives varient tellement qu'il serait impossible de les maintenir égales pendant un temps même très court.

Par contre, plus le contact de réglage est poussé à droite plus la précision de la mise en parallèle augmente, mais il est nécessaire d'obtenir une plus grande uniformité des deux fréquences pour provoquer la mise en parallele.

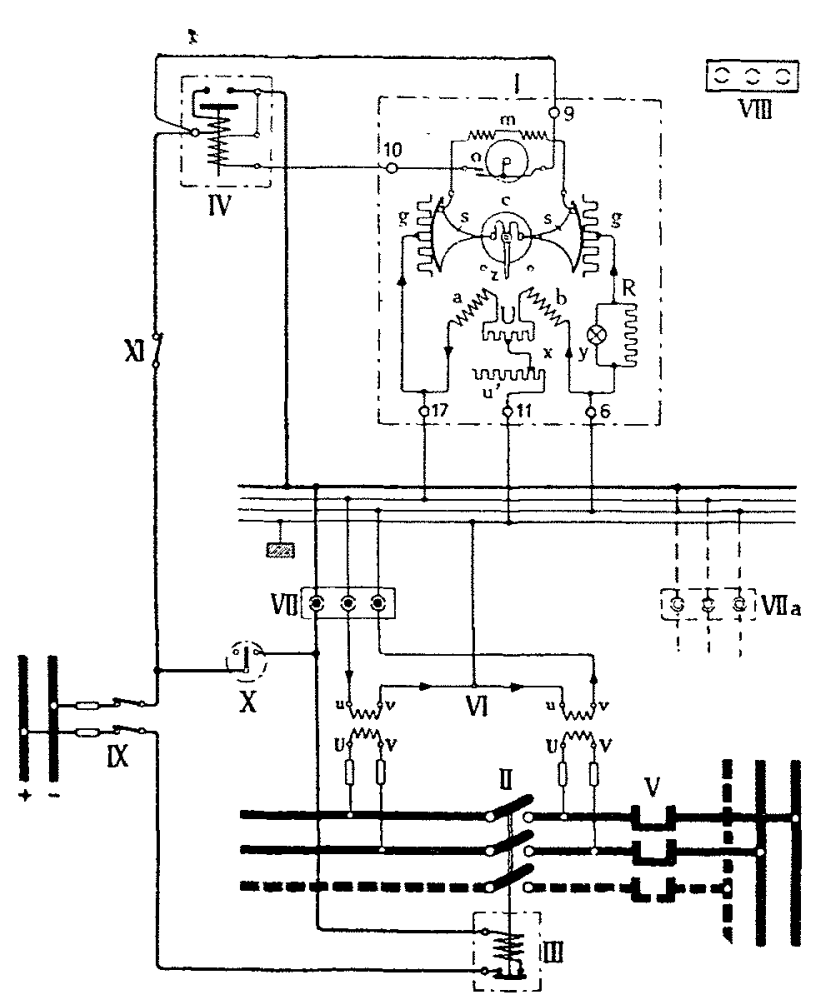

Fig. 8. - Disposition de l'appareil de mise en parallèle automatique pour une installation triphasée (commande à distance sans contact de fin de course).

$$
\begin{aligned}
\mathbf{I} & =\text { Appareil de mise en parallèle automatique. } \\
\text { II } & =\text { Interrupteur à huile. } \\
\text { III } & =\text { Commande à distance. } \\
\text { IV } & =\text { Relais intermédiaire avec bobine d'arrêt. } \\
\mathrm{V} & =\text { Sectionneur. } \\
\mathrm{VI} & =\text { Transiormateur de tension. } \\
\text { VII-VII a } & =\text { Commutateur à fiche pour Ies interrupteurs à lintile. } \\
\text { VIII } & =\text { Boîte a fiche vide. } \\
\text { IX } & =\text { Interrupteur du circuit auxiliaire. } \\
\mathrm{X} & =\text { Commutateur à main de la commande à distance. } \\
\mathrm{XI} & =\text { Interrupteur à bouton avec déclenchement refardé. }
\end{aligned}
$$

Ce dispositif de réglage permet d'adapter la précision de l'appareil aux conditions de service de l'entreprise, da façon à garantir la précision maximum correspondant à la meilleure uniformité de fréquence pouvant être obtenue avec une manœuvie de durée raisonnable. L'adaptation se fait une fois pour toutes à la première mise en service et l'appareil peut alors être plombé ou fermé par un cadenas pour éviter que le personnel de surveillance ne puisse modifier le réglage.

Il y a encore lieu de mentionner le dispositif de signalisation optique comportant un index oscillant combiné avec une lampe qui s'allumé périodiquenent el indiçue clairement si la vilesse de la machine à mettre en parallèle sur les barres doil être augmentée ou diminuée. L'appareil peut donc être employé comme syuchronoscope même lorsque, pour une raison quelconque, on ne veut pas provoquer la mise en parallèle automatique.

\section{APPLIC.ATIONs}

Dans une usine blectrique ou dans une sous-stalion, il suffit presque toujours d'installer un seul appareil de mise en parallèle automatique. On le branche au moyen de commutateurs à fiches sur les différentes unités et leurs interrupteurs à huile, suivant les besoins du service. La combinaison de ces commutateurs doit dans tous les cas ètre faite de telle façon qu'une fausse manoxuvre de l'homme de service ne puisse entraîner une mise en jarallèle inexacte. On ne doit pas, en effet, perdre de vue que l'un des grands avantages de cet appareil automatique est justement celui de permettre à un personnel inexpérimenté de réaliser une mise en parallèle absolument sûre et exacte.

Toutes les combinaisons de commutateurs qui n'excluent pas rigoureusement toute possibilité de fausse mancuvre pouvant provoquer une mise en parallèle inexacte doivent être rejetécs. Une de ces combinaisons dangereuses est celle consistant a se servir de transformateurs de tension branchés sur les barres onnibus lorsque l'usine présente plusieurs systèmes de barres travaillant indépendamment les unes des autres. Dans ce cas, il faut absolument prévoir un dispositif de verroullage pouvant ètre réalisé par des contacts auxiliaires montés sur l'axe des sectionneurs de façon à ne connecter à l'appareil de mise en parallèle que le transformateur de tension du système de barres sur lequel les sectionneurs ont été fermés (fig. 6).

Le couplage le plus sûr ét en mème temps le plus simple peut toujours être réalisé suivant le schéma.de la figure 3 ; on se sert pour l'alimentation de l'appareil de mise en parallèle de deux transformateurs de tension placés de chaque côté de l'interrupteur à huile à actionner. Comme on peut toujours pour alimenter l'appareil de mise en parallèle, se servir du transformateur do tension existant, sur leçuel sont déjà branchés les instruments de mesure, cette disposition de deux transformateurs de lension n'entraîne pas nécessairement une augmentation du nombre total des transformateurs. Il faut toutefois disposer ces transformateurs de façon à pouvoir réaliser cette combinaison. Ce ne sera donc que dans les cas assez rares où cette répartition des transformateurs ne serait pas possible sans frais importants que l'on se servira du dispositif indiqué sur la figure 6 .

Dans les installations où il n'y a qu'un seul systeme de barres, on pourra toujours se servir du transformateur de tension branché sur les barres.

Comme il a été dit précédemment, il est nécessaire que les transformateurs de tension alimentant l'appareil soient branchés de façon à produire, pendant l'identité de phase, l'allumage des lampes, c'est-ì-dire que la mise à terre devra se faire aux bornes différentes des transformateurs $(v, u)$. Si, par suite de l'installation antérieure des appareils, la mise à la terre des transformateurs de tension a déjà eté faite uniformément à la mème borne, d'où résulte donc le couplage à l'extinction des lampes, il faudra employer un transformateur-inverseur (fig. 7) pour rétablir le couplage à l'allumage, indispensable au bon fonctionnement de l'appareil.

Il existe un grand nombre de types diflérents de commandes à distance des interrupteurs à huile. Certaines commandes ont un 
contact de fin de course interrompant le circuit de la bobine d'enclenchement; d'autres ne possèdent pas ce dispositif. Afin d'assurer un bon fonctionnement de l'appareil de mise en parallèle, avec ces différents types de commandes, un certain nombre de combinaisons ont été étudiées. Les figures 3 et 6 représentent la disposition de commandes à distance munies d'un contact de fin de course. Comme le petit contact de l'appareil de mise en parallèle ne pourrait pas sans danger fermer et interrompre le courant généralement très important de la bobine d'enclenchement des commandes à distance, on se sert d'un relais intermédiaire IV. Ce petit contact ferme le circuit de la bobine de tension du relais intermédiaire. L'armature est attirée et le relais ferme le circuit de la bobine d'enclenchement de la commande à distance; en même temps, il court-circuite sa propre bobine de tension et met en circuit une bobine d'arrêt dont les enroulcments sont en série avec ceux de la commande à distance; cette bobine d'arrêt maintient la fermeture des contacts du relais intermédiaire. $\Lambda$ près la mise en parallèle, le contact de fin de course interrompt le circuit de la bobine d'enclenchement de la commande à distance, et par conséquent, celui de la bobine d'arrêt qui laisse alors retomber son noyau. Le circuit de la bobine de tcnsion est égalcment interrompu par le contact de fin de course, de sorte que, mème si le contact de l'appareil de mise en parallèle reste fermé, le relais intermédiaire n'est pas sous tension.

Si l'on voulait se servir de ce mème dispositif pour la commande à distance n'ayant pas de contact de fin de course, la bobine d'enclenchement resterait sous tension jusqu'à ce que l'homme de service en interrompe le circuit en retirant la fiche VII. Toutefois, cette bobine n'étant pas calculée pour supporter indéfiniment le courant d'enclenchement, elle s'échaufferait de façon dangereuse si l'interruption ne se faisait pas rapidement. On se servira donc avec avantage du dispositif indiqué sur la figure 8. Le relais intermédiaire IV ne possède pas alors de bobine d'arrêt, mais seulement un petit contact auxiliaire qui maintient les contacts principaux fermés lorsque la tension a été donnée par l'appareil de mise en parallèle. Un second relais XII, muni d'un dispositif d'amortissement, interrompt au bout d'un certain retard réglable le circuit de la bobine du relais intermédiaire, ce qui causc également la rupture du circuit de la bobine d'enclenchement de la commande à distance. Ce retard peut être réglọ à volonté de façon à laisser à celle-ci le temps suffisant pour fermer complètement l'interrupteur à huile. Ce dispositif possède encore un avantage, qui consiste en un blocage automatique du relais XII, qui né peut être libéré qu'à la main au moyen d'un bouton permettant la prochaine mise en parallèle. En cas de non fonctionnement pour cause de. dérangement dans l'interrupteur à huile ou dans sa commande à distance, on évite ainsi un "pompage " continu de cet interrupteur, car la commande à distance ne reçoit qu'une seule fois l'impulsion nécessaire à la fermeture de ce dernier.

L'appareil de mise en parallèle automatique avec les résistances du dispositif modificateur forme un tout complet, enfermé dans un boîtier pouvant être monté facilement sur un tableau. Les contacts et le système mobile sont enfermés hermétiquement derrière un couvercle avec cadre vitré qui évite toute détérioration. Les résistances placées derrière le tableau sont protégées par un couvercle en tôle perforée permettant le refroidissement. Toute usure est évitée et l'appareil ne nécessite pas plus d'entretien qu'un instrument de mesure.

\section{Aspect Général de l'Appareil}

Comme ses dimensions sont indépendantes de la puissance des groupes à mettre en parallèle, il n'existe qu'un seul type bobiné pour ètre branché sur un courant alternatif $2 \times 110$ volts. Le relais intermédiaire peut être livré pour courant continu ou courant alternatif. Afin de pouvoir calculer les bobines, il est nécessaire de connaitre le système de distribution et la tension de la source auxiliaire. Pour les dispositifs sur les figures 3 et 6 , il est nécessaire de connaitre également l'intensité du courant absorbé par la bobine d'enclenchement de la commande à distance, car cette intensité détermine la bobine d'arrêt du relais intermédiaire.

(S. V.) 